\title{
THE IDENTIFICATION OF LIFE HISTORY STRATEGY In A SHORT Projective TeST
}

\author{
Curtis S. Dunkel ${ }^{1}$, Steven C. Hertler ${ }^{2}$, Eugene W. Mathes ${ }^{1}$, and Tomás Cabeza de \\ Baca $^{3}$
}

'Western Illinois University, Macomb, Illinois, USA.

${ }^{2}$ Psychology Department, College of New Rochelle, New Rochelle, New York, USA. Psychology Department, College of Saint Elizabeth, Morristown, New Jersey, USA. Psychology Department, Caldwell University, Caldwell, New Jersey, USA. ${ }^{3}$ Division of Cardiology, University of California, San Francisco, California, USA.

c-dunkel@wiu.edu

\begin{abstract}
The covariance among a large cache of biopsychosocial individual differences (e.g., identity) has been explained by differences in life history $(\mathrm{LH})$ strategies. The current investigation was premised on the proposition that the LH nomological network may extend to individual differences on projective tests. In order to test this hypothesis, responses on a projective test of identity (the Twenty Statements Test), using three separate data sets (each from a different decade and spanning a period of 45 years), were scored using the LH Rating Form and in turn those scores were correlated with psychometric and biodemographic indices of LH strategy. The pattern of results was consistent with the hypothesis. The TST responses of slow LH strategy participants appeared more likely to include references to religion and family, while the TST responses of fast $L H$ participants seemed to refer to sex and drugs more often. These qualitative interpretations were consistent with the quantitative analyses, which were, in general, supportive of the predicted linear association between scored TST responses and the psychometric and biodemographic measures of LH strategy. In light of the findings, the relationship between LH strategy and identity is discussed. Further investigation on the association between LH strategy and projective tests appears warranted.
\end{abstract}

Keywords: Life history theory, twenty statements test, projective, $k$-factor. 


\section{INTRODUCTION}

The study of between-species differences in reproduction, growth, development, and senescence has identified covariation and integration among these facets across different species (Promislow \& Harvey, 1990). For example, species that produce a large number of offspring also tend to exhibit little parental investment, rapid growth and maturation, and early senescence. The coordination amongst these various characteristics is purported to represent a generalized reproductive strategy termed life history (LH) strategy. As opposed to a species with a fast LH strategy that allocates bioenergetic resources toward courting and mating (i.e., reproductive effort); species with a slow LH strategy allocate bioenergetic resources toward growth and development (i.e., somatic effort). In common, species exhibiting a fast $\mathrm{LH}$ compress their lifespans into shorter periods, and are thus marked by rapid gestation, maturation, and senescence. In turn, and by necessity, fast LH species provide scant parental care to their early and oft created progeny. Species exhibiting a slow life history show the opposite pattern in all respects, from protracted gestation to lavish parental care.

Recognizing that humans varied along a number of $\mathrm{LH}$ variables (e.g., gestation length) and related social behaviors (e.g., family structure), Rushton (1985) used LH theory to explain a cache of individual and group differences. Following in this tradition, Figueredo and colleagues (2004) factor analyzed 20 scales that were administered to a large representative sample of midlife adults. Although the scales were designed to measure an array of behavioral and cognitive individual differences such as familial support and self-directedness, factor analyses revealed a single common factor explained over $70 \%$ variance in the measures. This factor is called the $K$-factor. Since the initial discovery of the $K$-factor, research has added its broad nomological network (e.g., Patch \& Figueredo, 2017).

Identity appears to be included in the LH strategy nomological network. Several variants of the identity construct have been found to be associated with LH strategy such that ego-identity maturity is related to a slow LH strategy. In separate studies Dunkel and Sefcek (2009) found strong associations between various measures of identity and LH strategy. Likewise, Dunkel, Mathes, Harbke (2011) used several measures of identity to create a latent identity variable, referred to as identity consolidation (Schwartz, 2007), which in turn exhibited a strong association with a similarly created latent LH strategy variable. Lastly, narrative identity (Pals, 2006) as measured by participants' descriptions of their life's high and low points were rated by independent raters for participant LH strategy using the newly developed LH rating form (Dunkel et al., 2016). This last finding is important not only because it lent evidence to the validity of the LH rating form, but it also suggested that accurate judgments can be made by raters with only a minimal amount of information - a sample of the participants' autobiographical writing. In the current investigation we hoped to further explore these findings using participant responses on the Twenty Statements Test (TST; Kuhn \& McPartland, 1954).

The TST was designed to measure self-attitudes, yet can be clearly seen as a qualitative measure of identity (e.g., Vignoles, Regalia, Manzi, Golledge \& Scabini, 2006). Participants who are administered the TST are instructed to complete the sentence stem "I am. .." twenty times. Although Kuhn and McPartland (1954) state that the TST is not a projective test because it was not designed to reveal the subconscious, it 
shares characteristics with projective tests. The open structure of the TST allows participants to complete the twenty statements or sentence stems in any way they choose. Thus the TST is very similar to Loevinger's sentence stems (1998) used to measure ego development, which is recognized as a projective test (Lilienfeld, Wood \& Garb, 2000), and in fact the "I am ..." sentence stem is the $23^{\text {rd }}$ item in Loevinger's measure.

Thus, we explore the possibility that TST responses are associated with LH strategy. Specifically, it is predicted that LH form ratings based upon a participant's TST responses will be predictive of LH strategy. Narrowly such an association would add to the understanding of how identity is related to LH strategy. More broadly such an association would hold two additional important implications. First, it would suggest that LH strategy can be ascertained with some degree of accuracy with minimal information. Secondly, it would suggest that projective tests may reveal information concerning a respondent's LH strategy. This possibility is interesting as it suggests that we can start to explore the impact of LH strategy on the less overt/conscious aspects of cognition; the aspects of the mind projective tests were designed to measure.

While endeavoring to ascertain the relationship between the TST and LH strategy, we are cognizant of a recent discussion concerning the measurement of LH strategy (Copping, Campbell \& Muncer, 2014; Copping, Campbell, Muncer, \& Richardson 2016; Figueredo et al., 2015). At the heart of the discussion are the relative advantages and disadvantages of psychometric-Likert-type measures such as the $K$-factor, Mini- $K$ (Figueredo, Vásquez, Brumbach \& Schneider, 2004), and HKSS (Giosan, 2006), as opposed to biometric measures such as sexual behavior (e.g., age of sexual initiation, number of sexual partners). Most recently, however, Copping et al. (2016) suggest that both psychometric and biometric measures should be employed when possible. Hence, when examining the association between the TST and LH strategy we have attempted to use both psychometric and biodemographic measures of LH strategy. The term biodemographic is adopted to distinguish the measures used from both psychometric measures, but also from direct biological measures of LH strategy (e.g., skeletal age to measure pubertal maturation). That is, participants responded to questions about behaviors indicative of LH strategy, but no behaviors were observed nor actual physical measurements made.

Data from three data sets, collected in three separate decades $(1971,1985,2016)$ over a span of 45 years, were utilized in testing the hypotheses. In two data sets a psychometric measure of LH strategy was constructed, by either combining pre-existing scales or items using Figueredo and colleagues' work on the $K$-factor and Mini-K (2004) as a guide. In the third data set a measure specially designed to measure LH strategy, the Mini-K (Figueredo et al., 2004) was included. In all three data sets biodemographic items were included by asking participants about their sexual behavior (e.g., age of sexual initiation). 


\section{METHODS}

\section{Sample 1: Longitudinal Study of Generations (LSG)}

The LSG is a longitudinal study of multiple generations within 300 California families (Bengtson, 1971). For the current investigation, data from the subsample of grandchildren who were 18 or 19 years of age $(M=18.39, S D=.49)$ at the initial wave of data collection was used. There were 119 participants with TST data, 52 of whom were male and 67 of whom were female. The ethnic background of the participants, as described in the data file, were as follows: 87 White, 3 Hispanic, 2 Black, 2 Jewish, and 15 "other" or with missing data.

\section{TST and the LH rating form}

Participants were administered the TST. They were instructed to write down up to 20 responses to the question, "Who am I?" Three raters rated the TST responses using the $\mathrm{LH}$ rating form. One rater rated all of the responses, while another rater rated the responses of the 18 year-olds and the third rater rated the responses of the 19 year-olds. The internal consistencies for the three raters ranged from $\alpha=.69-.94$. The ratings for the two raters who rated the 18 or 19 year-olds were combined. The correlation between ratings was $r=.57$ with an intra class correlation using the average measures output of $r_{\mathrm{I}}$ $=.73$.

\section{Psychometric measure of LH strategy}

A unit-weighted $K$-factor was calculated by adding the standardized values (i.e., $z$-scores) from the following preformed scales described in the codebook and scales constructed from individual items: affectional solidarity mother (sample item: Overall, how well do you and your mother get along at this point in time?); affectional solidarity father; familism (sample item: Family members should give more weight to each other's opinions than to the opinions of outsiders); alienation (sample item: I often feel that there is very little meaning in my life); control (sample item: I have always been able to do whatever I set out to do in my own life.); religiosity as a sum of the $z$-scores of two items related to how often the participants attend religious services and their self-rated religiosity; and sexual attitudes as a sum of the $z$-scores of two items related to participants views of premarital sex. Note that "alienation" was reverse-scored prior to transforming and summing the $z$-scores.

\section{Biodemographic measure of LH strategy}

Answers to two questions were used to measure biodemographic LH strategy. One question, concerned the year participants "... started dating for the first time" and the second question concerned the year participants "...experienced their first sexual relations". An examination of the responses led to the removal of three data points for the dating question and four data points for the sexual relations question. For the dating item, one participant reported that their first date was a year later than the year data was acquired and two participants were determined to be outliers using the outlier labeling rule (how2stats, 2011); these participants reported that they had experienced their first date prior to the age of 10 . For the sexual relations question, one participant reported a 
year later than the year data was acquired and three participants were determined to be outliers using the outlier labeling rule; these participants reported that they had experienced their first sexual relations at or prior to 11 years of age. A total biodemographic (i.e., Sexuality factor) score was obtained by standardizing (transforming to $z$-scores) the response to the two items and summing the values.

\section{Sample 2: Computer Administered Panel Study}

The Computer Administered Panel Study (CAPS) was conducted between 1983 and 1988 at the University of North Carolina (Odum Archive, 1985). For each of those years a random sample of the undergraduate population was invited to participate in the CAPS project. The data analyzed in the current investigation is from the year 1985; the year the TST was administered.

Ninety-seven undergraduate students completed the TST. Of the 97, 49 were female and 48 were male. Eighty-four of participants were White and 13 were Black. The participants are described as being representative of the general student population with a range in age from 19 to $23(M=20.57, S D 1.16)$.

\section{TST and the LH rating form}

Instead of the standard 20 statements, participants were instructed to write down 10 statements in response to the question "Who am I?" Three raters read participants' response to the TST and then used the $\mathrm{LH}$ rating form to rate the participant. The internal consistency for the three raters ranged from $\alpha=.87-.95$ with an intra class correlation using the average measures output of $r_{I}=.70$.

\section{Psychometric measure of LH strategy}

A unit-weighted $K$-factor was calculated by adding the standardized values (i.e., $z$-scores) from the following preformed scales and items: sensation seeking (sample item: Choose one- I like "wild" uninhibited parties or I prefer quiet parties with good conversation); social support (sample item: I know someone who would loan me $\$ 100$ to help pay my tuition); internal locus of control (sample item: In my case, the good grades I receive are always the direct result of my efforts.); a single item assessing self-rated religiosity ( $1=$ More than average to $4=$ Not at all); a single item assessing attitudes toward sexual activity ( $1=$ Sex is not necessarily related to love or affection to $7=$ Sex is only acceptable as part of a serious loving relationship); two items assessing closeness to mother/father ( 1 = I can tell her/him almost anything to $5=\mathrm{I}$ do not feel free to tell her/him anything); and two items assessing value similarity with mother/father $(1=$ very similar values to $5=$ very different values). Note that when necessary, values were reverse scored (e.g., sensation seeking, religiosity, closeness with mother, closeness with father, value similarity with mother, value similarity with father) so that higher values were reflective of a higher $K$-factor score.

\section{Biodemographic measure of LH strategy}

Questions concerning sexual behavior were included in the CAPS Dating, Serious Relationships, and Sexuality module (1985). The questions and possible responses 
included the following: (Question 1) Have you ever been sexually active? (Responses) 1 $=$ Yes, have engaged in sexual intercourse; 2 = Yes, have engaged in petting, but not intercourse; 3 = No. (Question 2) Have you been sexually active in the last 6 months? (Responses) 1 = Yes, have engaged in sexual intercourse; 2 = Yes, have engaged in petting, but not intercourse; 3 = No. (Question 3) How many different partners have you engaged in sexual petting? (Responses) Anchored at $0=$ None and $5=$ Five or more. (Question 4) How many different partners have you engaged in sexual intercourse (Responses) Anchored at $0=$ None and $5=$ Five or more. (Question 5) How would you describe yourself and your sexuality? (Responses) $1=\mathrm{I}$ am not sexually active and have never had a sexual relationship; 2 = I am not sexually active now, but I have been sexually active in the past; 3 = I am involved in an exclusive relationship, and I am sexually active in that relationship; $4=\mathrm{I}$ date several people, and I am active with one or two of them; 5 $=\mathrm{I}$ date several people and I am sexually active with several of them; $6=\mathrm{I}$ am sexually active with many different people. A sexuality factor was constructed by standardizing (i.e., transforming the values to $z$-scores) the responses to the items and summing the values. Note that past sexual activity and sexual activity in the last six months were reverse scored prior to summing the values.

\section{Sample 3: Midwestern University Sample}

The sample was composed of 62 undergraduate students who were part of the undergraduate subject pool and who were participating in a larger study on individual differences in LH strategy. Of the 62 participants, 32 were male and 30 were female. Twenty-seven of the participants were White, 21 were Black, 11 were Hispanic, two participants responded other to the question on ethnicity, and one participant failed to respond. The participants were between the ages of 18 and $25(M=19.45 ; S D=1.44)$.

\section{TST and LH rating form}

Participants were administered a 20 -item TST which was subsequently scored by two raters using the LH rating form. The internal consistency for each of the raters was $\alpha=$. 93 and $\alpha=.95$. The correlation between raters was $r=.60$ with an intra class correlation using the average measures output of $r_{I}=.59$. Across the three data sets the intra class correlations are in the fair to good range (Cicchetti, 1994).

\section{Psychometric measure of LH strategy}

Participants completed an 18-item version of the Mini- $K$ (Figueredo et al., 2004; $\alpha=$. 68). Higher scores on the Mini- $K$ reflect a slower LH strategy. The original Mini- $K$ is 20 items, but because two items concern the participant's relationships that may be indicative of a fast LH strategy in a late adolescent/young adult sample an 18-item version omitting the two items was administered (Figueredo et al., 2006).

\section{Biodemographic measure of LH strategy}

Participants were asked four or five questions concerning their sexual behavior. The first question was, "With how many partners of the opposite sex have you had sex on one and only one occasion?" The second question was, "During your entire life, with how many partners of the opposite sex have you had sexual intercourse?" The third question was, 
"With how many partners of the opposite sex have you had sexual intercourse in the past year?" The fourth question was, "Have you had sexual intercourse (lost your virginity)?" If the answer to the fourth question was yes participants were then asked, "At what age did you have sexual intercourse for the first time?" A sexuality factor was constructed by standardizing (i.e., transforming the values to $z$-scores) the responses to the items and summing the values.

\section{RESULTS}

The three data sets were analyzed in succession. Correlations between the LH rating form scores with the LH psychometric and biodemographic measures were computed. Because in the LSG and the Midwestern University Sample the participants varied by age, partial correlations (controlling for age) were calculated. Partial correlations were used because the effect of age on each variable is controlled for. In the CAPS data all of the participants were the same age allowing for bivariate or zero-order correlations to be calculated. Sample responses for each data set can be seen in Table 1. Slow LH strategy participants appear more likely to have responses reflecting religiosity and the importance of family, while fast LH strategy participants have responses about sex and drugs or alcohol.

Table 1: Sample TST Responses.

Fast LH Responses

\begin{tabular}{l}
\hline LSG Sample: Good looking, Ugly, Decent build, Quick thinker, Drunkard (part-time), \\
Partier, Musician, Good lover, Mean if I need to be \\
CAPS Sample: Fun loving, Intelligent, Creative, Spontaneous, Crazy, Short-tempered, Lusty, \\
Straightforward, Hyperactive, Drunk. \\
Midwestern University Sample: 21, 63", A LEJA major, In a relationship, A sex addict, Not \\
into sports, Leaving the “the city in which the university is located”, A senior, Not into drugs, \\
An alcoholic, A lot of fun, In love, Super friendly, Outgoing, Religious, Bad at math, Great with \\
kids, A hard worker, Street smart, Fit.
\end{tabular}

\section{Slow LH Responses}

LSG Sample: Glad to be a girl, Want to be a Mother, Beginning to really understand people, Dissatisfied with my church, Happy with my husband, A little lazy, Sad to be so far from family, Not as worried what people think, Am very proud of my parents, Worried about parent's marriage, Like to sew my own clothes, Taught myself to crochet, Glad I started College Finally, My husband + I can talk about anything, Very honest + open with my husband, Have habits I wish I could overcome, Sleep too much, Tend to put things off, Kind to others, Honest + out right.

CAPS Sample: Caring about other people, Sweet and kind, Helpful, Lazy in the mornings, Love to talk to other people, 5'4" with short brown hair and brown eyes, A family person, Sort of independent, Likes to travel, Very close ties with where I grew up.

Midwestern University Sample: An athlete, Smart, Open minded, Strong, A country boy, A hard worker, Nice, A caring person, An American, A fighter, A person who never gives up, A man, A dog lover, Anti-woman beater, A fast thinker, A creative problem solver, A Christian, A son, A strong willed person, A rock for my friends and family. 
Quantitative results are presented in Table 2. Bivariate for the CAPS and partial correlations for the LSG and Midwestern University samples (controlling for age) between the $\mathrm{LH}$ rating form scores and the psychometric and biodemographic indices of LH strategy were calculated. As seen in Table 2, for the LSG the $K$-factor was significantly correlated with $\mathrm{LH}$ rating form scores, yet the sexuality factor was not significantly correlated with the LH rating form. For the CAPS sample, both the correlations with the $K$-factor and the sexuality factor were significant. Lastly, and also seen in Table 2, for the Midwestern University sample correlations between the LH rating form and the Mini- $K$ and both sexuality factor scores were significant.

Lastly, we computed correlations weighted by sample size between the LH rating form scores and the LH indices across studies. The average correlation between the LH rating form scores and psychometric LH combined across the LSG (using the partial correlation for the $K$-factor), CAPS (using the bivariate correlation for the $K$-factor), and the Midwestern University Sample (using the Mini-K) was .34. The average correlation between the LH rating form scores and biodemographic LH combined across the LSG (using the partial correlation for the sexuality factor), CAPS (using the bivariate correlation for the sexuality factor), and the Midwestern University Sample (using partial correlation for the sexuality factor not including the age of sexual initiation variable to increase sample size) was .29.

Table 2: Bivariate Correlations in the CAPS and Partial Correlations (Controlling for Age) in the LSG and Midwestern University Sample between the LH Rating Form Scores and Psychometric and Biodemographic Indices of LH Strategy.

\begin{tabular}{lc}
\hline \multicolumn{1}{c}{ Variable } & LH Rating Form \\
\hline LSG Sample: & $.28^{*}$ \\
K-factor & .20 \\
Sexuality factor & \\
CAPS Sample & $.40^{* * *}$ \\
K-factor & $.43^{* *}$ \\
Sexuality factor & $.33^{*}$ \\
Midwestern University Sample & $.30^{*}$ \\
Mini-K & $.36^{*}$ \\
Sexuality $t$-facor & \\
Sexuality factor including age of sexual & \\
initiation &
\end{tabular}

Note. ${ }^{*} p<.05 ; " p<.01, " p<.001$ 


\section{DISCUSSION}

\section{Summary of findings}

The hypothesis that LH theory could be used to explain individual differences on responses to the TST was tested. This proposition was tested using three data sets, across three decades. Participants' TST responses were scored by raters using the LH rating form and subsequently those ratings were correlated with psychometric and biodemographic measures of LH strategy. Consistent with hypothesis the $\mathrm{LH}$ rating form scores were positively correlated with psychometric LH strategy in each data set as measured by a $K$-factor in two data sets and the Mini- $K$ in the third data set. The average correlation between the $\mathrm{LH}$ rating form scores and the psychometric measures, across samples and weighted for sample size, indicated that the two variables shared a little over $10 \%$ of their variance.

The LH rating form exhibited significant positive correlations with biodemographic sexuality factors across two of the three data sets. The $\mathrm{LH}$ rating form score was not significantly correlated with the biodemographic sexuality factor in the LSG data set. We see two possible reasons for this null finding. First, the question about sexual initiation is ambiguous. Participants were asked if they had experienced their "first sexual relations" as a contrast to the more explicit wording used in the Midwestern University Sample in which participants were asked, "Have you had sexual intercourse (lost your virginity)?" Secondly, because of the secular trends in the age of marriage and attitudes toward premarital sex across the data sets, age of sexual initiation may be less closely tied to LH strategy in the LSG data set (data collected in 1971). For example, as seen in Table 1 the participant with the second highest LH rating form score in the LSG study has two TST responses that reference her husband. Presumably, the respondent has consummated the marriage with her husband.

\section{Conclusions, limitations and future directions}

Identity inquiry is a delicate matter. Precise questioning and operationalized definitions create uniformity among responses and increase generalizability across groups. On the other hand, this same approach stifles expression and is apt to obscure meaningful idiographic detail. At this early stage, in looking for life history themes, it seemed advisable to err on the side of idiographic detail. TST stems allow respondents valuable reign to establish their identity on self-chosen ground, while also providing narrative content that might further inform and refine future measurement strategies. Future research might follow Hertler, Krauss and Ward (2015) in using divergent measures of identity "in concert as a cooperative measurement strategy." For instance, an open-ended measure like the TST could be paired with a category specific measure informed by LH markers.

The actual identities of respondents are filtered through their response biases, imperfect measures, fallible raters and subjective content. For all of these shortcomings, one might expect LH strategy and identity to share more than the ten percent variance presently detected. Though we indeed suspect the existence of a more powerful relationship, we nonetheless predict a less than perfect relationship between LH strategy 
and identity even given perfect measurement. LH strategy impels psychological traits in certain directions. It may even be more correct to say that traits, conscientiousness for instance, are simply manifestations of LH strategy, retaining their distinct names and research programs for reasons of academic inertia. For many such variables, there is a direct and strong correlation derived of shared biological variance.

Identity is more conspicuously constructed. From an evolutionary perspective, its plasticity seems adaptive in that environmentally relevant content and successful persons can be imprinted and imitated. As such, it may then be important to know something of the dominant culture and subgroup; and to specifically know their aggregate LH strategy associated values, as a slow strategist in a fast dominated society may emulate fast LH themes even while expressing slow LH metrics. Putting such speculations aside, in future research, it might more certainly be productive to distinguish between identity content and identity theme. Such a distinction calls to mind the work of McAdams (1996) wherein seven features of identity narratives were outlined: (1) Emotional tone, (2) imagery, (3) theme, (4) ideological setting, (5) nuclear episodes, (6) imago (akin to Jungian personae), and (7) the generativity script. Roughly speaking and eschewing a detailed discussion, the first four of these seven, in that they seem to relate to one's core identity theme, may be most tightly constrained by life history, whereas the remaining three, in that they seem to relate to one's peripheral identity content, may be least tightly constrained by life history.

Aside from the topic of identity, the results suggest that projective tests supply relevant information about participant's LH strategy and that this information can be systematically assessed. This opens the door for further investigations into other projective tests and how they may relate to LH strategy. From this perspective, the importance of the findings lie in the consilience (Badcock, 1998; Wilson, 1998) of personality measures rooted in psychoanalytic models of personality with the metatheory of evolution (Buss, 1995). 


\section{REFERENCES}

Badcock, C. R. (1998). PsychoDarwinism: The new synthesis of Darwin and Freud. In C. B. Crawford \& D. L. Krebs (Eds.), Handbook of evolutionary psychology: Ideas, issues and applications (pp. 457-483). Mahwah, New Jersey: Erlbaum.

Bengtson, Vern L. Longitudinal Study of Generations, 1971, 1985, 1988, 1991, 1994, 1997, 2000 [California] [Computer file]. ICPSR22100-v2.

Bibliographic Citation: Ann Arbor, MI: Inter-university Consortium for Political and Social Research [distributor], 2009-05-12. DOI

Buss, D. M. (1995). Evolutionary psychology: A new paradigm for psychological science. Psychological Inquiry, 6(1), 1-30. DOI

Cicchetti, D. V. (1994). Guidelines, criteria, and rules of thumb for evaluating normed and standardized assessment instruments in psychology. Psychological Assessment, 6(4), 284-290. DOI

Computer Assisted Panel Study (CAPS). (1985). Dating, Serious Relationships, and Sexuality (CAPS-DATING module) 2009, "Dating, Serious Relationships, and Sexuality (CAPSDATING module)", hdl:1902.29/CAPS-DATING, UNC Dataverse, V1.

Copping, L. T., Campbell, A., \& Muncer, S. (2014). Psychometrics and life history theory: The structure and validity of the High K Strategy Scale. Evolutionary Psychology, 12(1), 200222. DOI

Copping, L. T., Campbell, A., Muncer, S., \& Richardson, G. B. (2016). The psychometric evaluation of human life histories: A reply to Figueredo, Cabeza de Baca, Black, Garcia, Fernandes, Wolf, and Woodley (2015). Evolutionary Psychology, 15(1), 1-14. DOI

Dunkel, C. S., Brown, N. A., Mathes, E. W., Summerville, L., Kesselring, S. N., \& Colclasure, R. (2016). Testing the Life History Rating Form. Evolutionary Behavioral Sciences, 10(3), 202-212. DOI

Dunkel, C.S., Mathes, E., \& Harbke, C.R. (2011). Life history strategy, identity consolidation, and psychological well-being. Personality and Individual Differences, 51(1), 34-38. DOI

Dunkel, C. S., \& Sefcek, J. A. (2009). Eriksonian lifespan theory and life history theory: An integration using the example of identity formation. Review of General Psychology, 13(1), 13-23. DOI

Figueredo, A. J., Vásquez, G., Brumbach, B. H., Schneider, S. M., Sefcek, J. A., Tal, I. R., Hill, D., Wenner, C. J. \& Jacobs, W. J. (2006). Consilience and life history theory: From genes to brain to reproductive strategy. Developmental Review, 26(2), 243-275. DOI

Figueredo, A. J., Vásquez, G., Brumbach, B. H., \& Schneider, S. M. R. (2004). The heritability of life history strategy: The $\mathrm{K}$-factor, covitality, and personality. Social Biology, 51(3-4), 121-143. DOI

Giosan, C. (2006). High-K strategy scale: A measure of the high-K independent criterion of fitness. Evolutionary Psychology, 4(1), 394-405. DOI

Hertler, S. C., Krauss, H., \& Ward, A. (2015). Assessing diachronic reasoning: exploratory measures of perceived self-change in young adults. Psychological Reports, 116(1), 176193. DOI

howwstats. (2011, September 8). The right way to detect outliers - Outlier Labeling Rule. [Video file]. Retrieved from https://www.youtube.com/watch?v=WSflSmcNRFI

Kuhn, M.H. \& McPartland, T.S. (1954). An empirical investigation of self-attitudes. American Sociological Review, 19(1), 68-76. DOI 
Lilienfeld, S. O., Wood, J. M., \& Garb, H. N. (2000). The scientific status of projective techniques. Psychological Science in the Public Interest, 1(2), 27-66. DOI

Loevinger, J. (1998). Technical foundations for measuring ego development: The Washington University Sentence Completion Test. Mahwah, New Jersey: Erlbaum. DOI

McAdams, D. P. (1996). Personality, modernity, and the storied self: A contemporary framework for studying persons. Psychological Inquiry, 7(4), 295-231. DOI

Odum Archive. (1985). Computer Assisted Panel Study (CAPS) [Data files and codebooks]. Retrieved from https://dataverse.unc.edu/dataverse/caps.

Pals, J. L. (2006). Narrative identity processing of difficult life experiences: Pathways of personality development and positive self-transformation in adulthood. Journal of Personality, 74(4), 1079-1110. DOI

Patch, E. A., \& Figueredo, A. J. (2017). Childhood stress, life history, psychopathy, and sociosexuality. Personality and Individual Differences, 115, 108-113. DOI

Promislow, D. E. L., \& Harvey, P. H. (1990). Living fast and dying young: A comparative analysis of life-history variation among mammals. Journal of Zoology, 220(3), 417-437. $\underline{\text { DOI }}$

Rushton, J. P. (1985). Differential K Theory: the sociobiology of individual and group differences. Personality and Individual Differences, 6(4), 441-452. DOI

Schwartz, S. J. (2007). The structure of identity consolidation: Multiple correlated constructs or one superordinate construct? Identity, 7(1), 27-49. DOI

Vignoles, V. L., Regalia, C., Manzi, C., Golledge, J., \& Scabini, E. (2006). Beyond self-esteem: Influence of multiple motives on identity construction. Journal of Personality and Social Psychology, 90(2), 308-333. DOI

Wilson, E. O. (1998). Consilience: The unity of knowledge. New York: Knopf, Inc. 